\title{
Segregation of mutations in arylsulphatase E and correlation with the clinical presentation of chondrodysplasia punctata
}

The Murdoch Institute for Research into Birth Defects, The Royal Children's Hospital, Flemington Road, Parkville, Melbourne, Victoria 3052,

Australia

L J Sheffield

A H Osborn

W M Hutchison

$S$ M Forrest

H-H M Dahl

The New Children's Hospital, Westmead, NSW, Australia D O Sillence

Adelaide Women's and Children's Hospital, SA, Australia S J White

Correspondence to: Dr Sheffield.

Received 11 December 1997 Revised version accepted for publication 27 April 1998

\author{
L J Sheffield, A H Osborn, W M Hutchison, D O Sillence, S M Forrest, S J White, \\ H-H M Dahl
}

\begin{abstract}
Sixteen males and two females with symmetrical (mild) type of chondrodysplasia punctata were tested for mutations in the $\mathbf{X}$ chromosome located arylsulphatase $D$ and $E$ genes. We identified one nonsense and two missense mutations in the arylsulphatase $E$ gene in three males. No mutations were detected in the arylsulphatase D gene. Family studies showed segregation of the mutant genes establishing $X$ linked inheritance for these families. Asymptomatic females and males were found in these studies. The clinical presentation varies not only between unrelated affected males, but also between affected males within the same family. We also conclude that clinical diagnosis of chondrodysplasia punctata in adults can be difficult. Finally, our results indicate that brachytelephalangy is not necessarily a feature of $X$ linked symmetrical chondrodysplasia punctata.

$(\mathcal{O}$ Med Genet 1998;35:1004-1008)
\end{abstract}

Keywords: chondrodysplasia punctata; arylsulphatase; mutations

Chondrodysplasia punctata (CDP) refers to a group of bone dysplasias characterised by punctate changes in infants seen radiologically around developing epiphyses. A number of distinct types can be recognised and the cause can be primary, usually genetic, or secondary owing to side effects of drug ingestion during pregnancy or a consequence of certain storage diseases. ${ }^{12}$ We have classified 93 primary cases radiologically and 83/93 cases fell into one category, the symmetrical type. ${ }^{34}$ This group was initially called mild (Sheffield type). ${ }^{2-5}$ More recently, the term symmetrical chondrodysplasia punctata (SCDP) has been proposed. ${ }^{4}$ Some of these cases have terminal phalangeal hypoplasia (brachytelephalangy) and could be classified as brachytelephalangic CDP, which is thought to be an $\mathrm{X}$ linked recessive form of the disease (MIM302940). ${ }^{6}$ Our previous clinical study could not differentiate between $\mathrm{X}$ linked recessive, symmetrical CDP (CDPX), and brachytelephalangic CDP, all of which had symmetrical radiological punctate changes or shortening of long bones. ${ }^{34}$ As symmetrical CDP was shown in our study to have a sex ratio of two males to one female ${ }^{3}$ and to be the most common type of CDP, it is possible that many cases of CDP are X linked.
The gene(s) causing CDPX was mapped to $\mathrm{Xp22.3}$ in individual patients classified as symmetrical or brachytelephalangic CDP. ${ }^{78}$ This led to the isolation and characterisation of a cluster of three arylsulphatase genes (ARS-D, ARS-E, and ARS-F). ${ }^{9}$ Missense mutations in the ARS-E gene were present in five patients with CDPX, strongly suggesting that CDPX is caused by arylsulphatase $\mathrm{E}$ deficiency. No families were studied however. We have tested 16 males and two females who have symmetrical CDP for the presence of mutations in the coding regions of the ARS-D and ARS-E genes. We report the clinical findings, identification, and segregation of three novel mutations in the ARS-E gene in three families with symmetrical CDP.

\section{Materials and methods}

PATIENTS

The 16 males and two females with symmetrical SCDP analysed in this study all had the diagnosis made by showing puncta radiologically. They had been part of a previous study of the classification of SCDP by Sheffield $e t a l^{34}$ and six of these males had been analysed, but no mutations found, by SSCP for ARS-D and ARS-E mutations in the initial study that defined ARS-E as the causative mutation in CDP. ${ }^{9}$ Brachytelephalangic CDP was not classified separately from SCDP because we had previously concluded that they were the same entity. ${ }^{3}$ They were classified radiologically and by exclusion of a history of exposure of the fetus to phenytoin or warfarin during pregnancy or by exclusion of any other syndromic diagnosis. There were six cases with a known family history.

\section{DNA ANALYSIS}

DNA extraction from blood and cultured fibroblasts was carried out as published by Blok et al. ${ }^{10}$ Individual exons were PCR amplified by use of intron specific primers (G Meroni, unpublished data). Fragments were labelled internally with $\left[\alpha-{ }^{33} P\right]-d A T P$ during PCR amplification.

Single strand conformation polymorphism (SSCP) detection was performed on the amplified exon fragments basically as described by Orita et al. ${ }^{11}$ Five $\mu$ l of the PCR product were diluted in $45 \mu \mathrm{l}$ of denaturing buffer $(90 \%$ formamide, $0.5 \times \mathrm{TBE}, 0.25 \%$ bromophenol blue, $0.25 \%$ xylene cyanol). The DNA was denatured by heating to $95^{\circ} \mathrm{C}$ for five minutes and then kept on ice until loading on $6 \%$ non-denaturing polyacrylamide gels with 

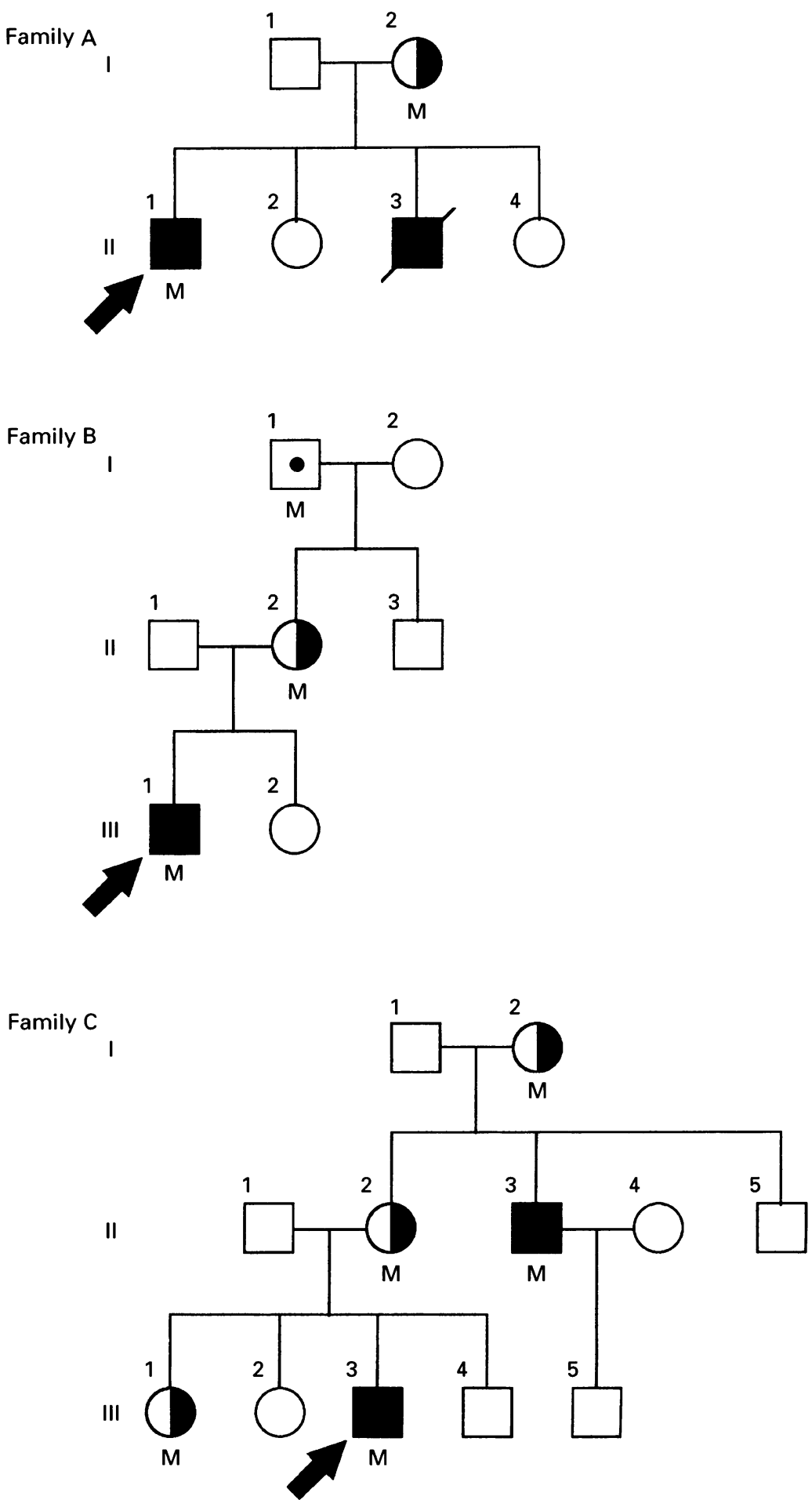

Figure 1 Pedigrees of families $A, B$, and $C$. The probands are indicated with an arrow and $M$ indicates the presence of the mutation.

or without $10 \%$ glycerol. Electrophoresis was carried out at $30 \mathrm{~W}$. The gels containing glycerol were run for approximately five hours at $4^{\circ} \mathrm{C}$, and the gels without glycerol were run for approximately three hours at room temperature. Gels were dried and exposed to Kodak Biomax MR film.

PCR fragments were purified from $3 \%$ NuSieve agarose gels using a $200 \mu$ l plugged tip purification method. ${ }^{12}$ The DNA was sequenced as described by Blok et $a l^{10}$ using a Sequenase version 2.0 kit (USB).
Numbering of nucleotides and amino acids are as in Franco et al. $^{9}$

\section{Results}

Genomic DNA was isolated from 16 male and two female patients diagnosed with chondrodysplasia punctata and exons in the ARS-D and ARS-E genes were tested for nucleotide changes by SSCP analysis. In six of the males some exons had been analysed for ARS-D and ARS-E mutations in a previous study. ${ }^{9}$ When band shifts were detected on the SSCP autoradiographs, the fragment was further analysed by DNA sequencing. No DNA sequence information was available for the ARS-F gene, and we were therefore unable to include this gene in the present study. Nucleotide changes that did not alter an amino acid or were subsequently detected in samples from unaffected controls were classified as polymorphisms. However, three male patients had nucleotide changes in the ARS-E gene which were considered to be causative mutations. All mutations were novel, single nucleotide changes and located in exons 4,6 , and 10 , respectively. DNA sequencing showed that the exon 4 and 6 mutations were missense mutations and the exon 10 mutation introduced a premature stop codon. The families of these patients were studied to document segregation of the mutation, with two of the three families having a previously known family history (fig 1). The mutations were found in three of the 10 male patients not studied previously and represent a $30 \%$ detection rate of mutations.

FAMILY A

The male proband (fig 1) was first seen at 2 years of age because of maternal concern about his facial appearance. He had a depressed nasal tip, noted from birth, and had short fingers. On examination, maxillary hypoplasia was noted with a flattened nasal tip and short columella characteristic of chondrodysplasia punctata. $\mathrm{He}$ was on the 50th height centile and had short terminal phalanges of the hand (brachytelephalangy). Clinically he was thought to be typical of brachytelephalangic chondrodysplasia punctata. Skeletal survey showed small, indefinite punctate calcifications on the medial side of the right ankle and hypoplasia and dysplasia of the calcaneal and talar bones. Minor dysplastic changes were present at the medial distal femoral ossification centres. The distal phalanges in both hands were short and dysplastic. His mother was asymptomatic and was of normal height with normal facies. The family history showed that a male sib had been stillborn with a diagnosis of chondrodysplasia punctata and anoxia. A skeletal survey in this sib showed punctate calcification around the femoral head and along the vertebrae. Histology of the femoral head showed abnormal matrix morphology and staining with clustering of the chondrocytes into islands surrounded by matrix with patchy reduction in proteoglycan (alcian blue positive and safronin $O$ positive staining). In the femoral head there was one large lesion surrounded by numerous smaller lesions. The vertebral body examined 
G317R

N F L G R S L H $R$

L Y G D N E M D W V GR

L Y G D N V E B M D W V V R

L Y G D N V B E M D W L I G K

ARS-C DFAG S Q B G VY Y D A VE E D W V G Q

ARS-B DFIQDKNRH HYAMVSLMDEVGN

ARS-A SF A ER S R G

Gal6s PFIGTSQRG RYGDAVREIDDSIGK G6S QFLD N A F R RWQTLLS - VDDLVER VDFQRKIRQ SYFASVSYIDTQVGR$$
\text { - I TFAKLIKD - Q G Y S }
$$

ARS-B

$$
\text { - KILPQLIRB - AGYT THAVGRW }
$$

ARS-A

Gal6s

$$
\text { - VTVAEVLAA-RGYL TGMAGKW } 124
$$

Figure 2 Mutations and protein homology among human sulphatases. ARS $-A,-B,-C$, $-D$, $-E$ : arylsulphatase $-A,-B,-C,-D,-E ;$ Gal $6 S: N$-acetylgalactosamine-6-sulphatase; G6S: glucosamine-6-sulphatase; IDS: iduronate sulphatase. The two mutant chains are shown (G317R and G137A) and the mutated glycines are indicated in bold.

was most abnormal with patchy, abnormal staining of cartilage and matrix, and mixed, dense, and loose connective tissue. Other postmortem findings were haemorrhagic thymus, petechial haemorrhages over the visceral pleura and pericardium, normal body proportions between limbs and trunk, and short looking hands. Cerebrospinal fluid was heavily blood stained. There was calcification of the great vessels.

A $G$ to A change was found in exon 6 of the ARS-E gene at nucleotide position 1016 , causing a glycine to arginine substitution at amino acid 317 (G317R). The nucleotide change results in the abolition of a $D s a I$ restriction endonuclease cleavage site. PCR amplification of genomic DNA using the exon 6 specific primers as described in Materials and methods gave a 271 bp band. When control DNA was analysed, this PCR fragment is cleaved by $D s a I$ to give 102 and 169 bp bands. The PCR product from this patient was not cleaved by $D s a I$.

The mother of patient $A$ was shown to be heterozygous for the mutation by DNA sequence analysis and PCR/DsaI analysis. This base change was not seen in 33 other people diagnosed with SCDP or in 17 normal control DNAs.

\section{FAMILY B}

The proband (fig 1) was seen at 3 months of age following $x$ rays showing punctate changes and a flat nose. He was one of the 23 patients described in the initial paper of Sheffield et at that defined the mild (symmetrical) type of
CDP. On examination he had typical facial features of chondrodysplasia punctata and was just below the 3 rd centile for length. At 9 months of age he was on the 25 th length centile and was between the 50 th and 75 th centile by the age of 11 years. He was last seen at the age of 20 years and was $184 \mathrm{~cm}$ tall (90th centile) and his nose was only a little flat. He had left school at year 9 because of poor school performance and had had several unskilled and semi-skilled jobs. His mother had a normal facies and was of normal height, as were her own parents.

Following SSCP analysis we identified a $\mathrm{G}$ to C change in ARS-E exon 4 (at nucleotide 477) of the male proband. This results in a glycine to alanine substitution at amino acid 137 (G137A) (fig 2). The asymptomatic maternal grandfather also carries this mutation and the clinically normal mother is heterozygous for the mutation. DNA sequence analysis failed to detect the base change in 18 chromosomes from other CDP patients and 22 chromosomes from unaffected controls.

FAMILY C

The proband (fig 1) was born after a normal pregnancy and delivery. He was noticed to have severe nasal hypoplasia and a very flat nose. As a consequence he had difficulties in breathing. Other dysmorphic signs noted were micrognathia, PDA, small VSD, and mild pulmonary stenosis. Skeletal films confirmed chondrodysplasia punctata with widespread puncta seen in the tarsal bones, femoral neck, and in the distal phalanges. There were sagittal clefts in the vertebrae. Chromosome studies showed a normal $46, \mathrm{XY}$ karyotype. He was found to have a mild to moderate bilateral sensorineural hearing loss of unknown cause, but had viral meningitis at the age of 3 months. His height and weight were on the 3 rd centile at 26 months of age. He was first examined at 16 years 10 months of age. His height was $170 \mathrm{~cm}$ (10th-25th centile). He had maxillary hypoplasia and a nose with the typical appearance (short columella) of chondrodysplasia punctata with a high nasal bridge owing to a silicone implant. He had short terminal phalanges (brachytelephalangy) and a short looking neck. Learning problems were experienced at school and he required special help.

There was a maternal uncle who had been very short as a child, had a similar facial appearance, and had terminal phalangeal hypoplasia. When seen as an adult his nose had grown considerably and he had a near normal appearance.

Sequence analysis of the proband showed a $G$ to $A$ nucleotide change in codon 581 in ARS-E exon 10, which changed the codon for tryptophan to a TGA stop codon. The mutation was also present in the mother, a sister, a maternal grandmother, and the maternal uncle mentioned above.

\section{POLYMORPHISMS}

SSCP and sequence analysis identified several polymorphisms in the ARS-E gene. These were $T$ to $G$ at nt $562\left(\mathrm{His}^{165}\right), G$ to $A$ at nt 853 
$\left(\mathrm{Thr}^{262}\right), \mathrm{G}$ to $\mathrm{A}$ at nt 1337 ( $\mathrm{Gly}^{424}$ to Ser), C to $\mathrm{T}$ at nt $1759\left(\mathrm{Asn}^{564}\right)$, and $\mathrm{G}$ to $\mathrm{A}$ at nt 1795 $\left(\right.$ Pro $\left.^{576}\right)$. In all samples analysed, we detected the sequence GAC at nt 572, suggesting an Asp ${ }^{168}$ instead of a Glu.

\section{Discussion}

Franco et al identified three arylsulphatase genes (D, E, and $F$ ) in the chromosome $\mathrm{Xp22.3}$ region. They suggested that $\mathrm{X}$ linked recessive chondrodysplasia punctata (CDPX) was caused by mutations in ARS-E on the basis of finding mutations in $5 / 27(19 \%)$ unrelated male CDP patients (brachytelephalangic and symmetrical CDP types) who were found to have missense mutations. We have similarly found that patients characterised as SCDP clinically have been found to have mutations in the ARS-E gene in $3 / 10$ males (30\%). Recently, a cysteine to tyrosine substitution at position 492 was reported in a patient with CDPX. ${ }^{13}$

The G317R mutation (family A) has not previously been reported. It is present in a part of the ARS-E protein that is highly homologous to related sulphatases (fig 2). A glycine residue is also found in this position in ARS-A, -C, and -D. The glycine to arginine substitution is likely to have consequences for protein structure by changing a polar amino acid to a basic amino acid. The proband in the family had the classical mild type of SCDP (normal height, brachytelephalangy, and normal intelligence with flattened nose as the only clinical manifestation).

The G137A mutation (family B) is also novel, but a glycine to valine mutation at the same amino acid (G137V) has been described. ${ }^{9}$ The glycine is present in related human sulphatases and located in a highly conserved region in these enzymes (fig 2 ). The mutation site has been described as crucial for the secondary structure of the arylsulphatases as judged by a severe impairment of processing and stability of arylsulphatase $B$ in a patient with Maroteux-Lamy syndrome. In this patient, a G137V mutation was observed in the arylsulphatase B gene. ${ }^{14}$ It is thus surprising that the phenotype in this family is so mild with a proband on the 90th centile for height without brachytelephalangy and a transmitting grandfather who is asymptomatic.

The codon 581 A to $G$ mutation in exon 10 (family C) introduces a translation stop codon, resulting in premature termination of the protein by nine amino acids. This is the first example of a nonsense mutation in the ARS-E gene causing CDP. Clinically the proband is the most severely affected of the three patients described here (very flat nose, short stature, other dysmorphic signs, and sensorineural deafness). This suggests an important role of the C-terminal end of the protein chain in enzyme stability or function. This family is also the first family described in which there is segregation of an ARS-E mutation which correlates with affected phenotype. However, the affected uncle with the same mutation was not as severely affected.

The genetics of CDP is complicated. The ARS-D, ARS-E, and ARS-F genes are located approximately $150 \mathrm{~kb}$ from the pseudoautosomal boundary in the $\mathrm{X}$ specific region, but they escape normal $\mathrm{X}$ chromosome inactivation. ${ }^{9}$ In all of our three families there were females of normal stature with normal facies who were shown to transmit the mutation, suggesting recessive inheritance. The sex ratio of cases seen is 2:1 (male to female), ${ }^{3}$ although we only studied two affected female patients in the current study. There is an $\mathrm{X}$ linked dominant form of CDP but this is clearly separated from SCDP on clinical grounds and is thought to have a separate locus at Xq28. ${ }^{15}$

The mode of inheritance of SCDP is also not clearly known. Maroteux proposed that brachytelephalangic CDP was $\mathrm{X}$ linked. ${ }^{68}$ The probands in family $A$ and $C$ did have brachytelephalangy, whereas family B did not. We found in a previous clinical study that the presence of brachytelephalangy merely related to whether puncta were present in the terminal phalanges and one could not differentiate patients with or without brachytelephalangy on other clinical features. Furthermore, there were subjects who had short terminal phalanges on some digits and not on others. The fact that family B did not have brachytelephalangy shows that this is not necessarily a feature of CDPX or of mutations in the ARS-E gene and supports the above conclusion that symmetrical CDP encompasses the subgroup of brachytelephalangy.

We have previously suggested that SCDP is a useful description of cases of CDP that appear clinically indistinguishable. ${ }^{3}$ These cases have been labelled as mild, ${ }^{5}$ brachytelephalangic, ${ }^{6}$ and $\mathrm{X}$ linked recessive with chromosomal deletion. In lumping all our cases together as $\mathrm{SCDP}^{4}$ on clinical and radiological grounds we may be combining cases resulting from more than one gene locus. However, our finding of three mutations in the 10 male patients who were not analysed for mutations in the study of Franco et al suggests that at least $30 \%$ of cases of SCDP are $\mathrm{X}$ linked and that mutations in the ARS-E gene are a major cause of SCDP. This in turn establishes $\mathrm{X}$ linkage as an important mode of inheritance. Most cases of SCDP do not have a known family history, so finding an ARS-E mutation enables genetic counselling regarding the inheritance mode.

We have identified mutations in ARS-E in $30 \%$ of our patients. In the previous study, mutations were found in approximately $20 \%$ of patients. ${ }^{9}$ The two studies found that $50 \%$ of identified mutations in the ARS-E gene were in exon 4. Furthermore, all mutations found in the two studies were different. SSCP analysis would be expected to detect a much higher proportion of mutations if they are present and this raises the possibility that some of the patients have SCDP not resulting from ARS-E mutations. We have tested 16 male and two female patients for nucleotide changes in the ARS-D gene, but no mutations were found. The ARS-F gene was not analysed. It is therefore possible that mutations in other genes are responsible for a subset of SCDP cases.

It is also important to note the grandfather in family B, who had a normal facies and stature 
and transmitted the mutation. It is not clear why the gene defect manifested itself only in the grandson. However, it may be because the nose grows out with age and adults with the condition can be much less confidently diagnosed. In fact, our previous clinical study predicted that there would be some asymptomatic "affected" parents as so few patients had a clear cut $\mathrm{X}$ linked family history. This missense mutation is associated with the mildest phenotype and this is shown in the proband (typical facies and initial growth retardation resulting in an adult of normal height). In contrast, the stop mutation in family $\mathrm{C}$ is associated with a more severe phenotype.

We have shown segregation of ARS-E mutations for the first time and shown that asymptomatic females and males can transmit the mutations. We identified ARS-E mutations in $30 \%$ of males with SCDP, suggesting that some patients with SCDP have mutations in other genes. We have also tested the ARS-D gene, but as in the earlier study ${ }^{9}$ no mutations were detected. However, the finding of three families where ARS-E mutations segregate with SCDP allows further definition of the phenotype, prognosis, and molecular pathology of SCDP.

We thank A Ballabio, B Franco, and G Meroni for making DNA sequence data available to us before publication, $A$ Holloway for
technical assistance, and $R$ Williamson for helpful discussions technical assistance, and $\mathbf{R}$ Williamson for helpful discussions
and support. This work was supported in part by an Institute and support. This work was supported in part by an Institute Grant from the National Health and Medical Research Council
(NH\&MRC) of Australia. H-HMD is an NH\&MRC Senior (NH\&MRC) of
Research Fellow.
1 Spranger JW, Opitz JM, Bidder U. Heterogeneity of chondrodysplasia punctata. Humangenetik 1971;11:190212.

2 Wulfsberg EA, Curtis J, Jayne $\mathrm{CH}$. Chondrodysplasia punctata: a boy with $\mathrm{X}$-linked recessive chondrodysplasia punctata due to an inherited $\mathrm{X}-\mathrm{Y}$ translocation with a current classification of these disorders. Am F Med Genet 1992; 43:823-8.

3 Sheffield LJ, Halliday JL, Danks DM, et al. Clinical, radiological and biochemical classification of chondrodysplasia punctata. Am 7 Hum Genet 1989;45.A64.

4 Sheffield LJ, Halliday JL, Jensen F, Danks DM. To lump or split types of chondrodysplasia punctata? Proc Greenwood Genetic Centre 1991;10:112-13.

5 Sheffield LJ, Danks DM, Mayne V, Hutchinson AL. Chondrodysplasia punctata - 23 cases of a mild and relatively drodysplasia punctata - 23 cases of a mild
common variety. $₹$ Pediatr 1976;89:916-23.

6 Maroteaux P. Brachytelephalangic chondrodysplasia punctata: a possible X-linked recessive form. Hum Genet 1989;82:167-70.

7 Ballabio A, Parenti G, Carrozzo R, et al. Isolation and characterization of a steroid sulfatase cDNA clone: genomic deletions in patients with X-chromosome-linked ichthyosis. Proc Natl Acad Sci USA 1987;84:4519-23.

8 Petit C, Melki J, Levilliers J, et al. An interstitial deletion in $\mathrm{Xp} 22.3$ in a family with X-linked recessive chondrodyspla sia punctata and short stature. Hum Genet 1990;85:247-50.

9 Franco B, Meroni G Parenti G, et al A cluster of sulfatase genes on $\mathrm{Xp} 22.3$ : mutations in chondrodysplasia punctata (CDPX) and implications for warfarin embryopathy. Cell 1995;81:15-25.

10 Blok RB, Thorburn DR, Thompson GN, Dahl HH. A topoisomerase II cleavage site is associated with a novel topoisomerase II cleavage site is associated with a novel

11 Orita M, Suzuki Y, Sekiya T, Hayashi K. Rapid and sensitive detection of point mutations and DNA polymorphism using the polymerase chain reaction. Genomics 1989;5:8749.

12 Dean $\mathrm{AD}$, Greenwald JE. Use of filtered pipet tips to elut DNA from agarose gels. BioTechniques 1995;18:980.

13 Parenti G, Buttitta P, Meroni G, et al. X-linked recessive chondrodysplasia punctata due to a new point mutation of the ARSE gene. Am $\mathcal{F}$ Med Genet 1997;73:139-43.

14 Wicker G, Prill V, Brooks D, et al. Mucopolysaccharidosis VI (Maroteaux-Lamy syndrome). An intermediate clinical phenotype caused by substitution of valine for glycine at position 137 of arylsulfatase B. 7 Biol Chem 1991;266: 21386-91.

15 Ropers HH. Personal communication. OMIM (TM) Online Ropers HH. Personal communication. OMIM (TM) Online Baltimore: Johns Hopkins University, WWW URL: http:// Baltimore: Johns Hopkins University
www.ncbi.nlm.nih.gov/Omim/ 1987 . 